\title{
Individual differences in perceptual space for tactile textures: Evidence from multidimensional scaling
}

\author{
MARK HOLLINS, SLIMAN BENSMAÏA, KRISTIE KARLOF, and FORREST YOUNG \\ University of North Carolina, Chapel Hill, North Carolina
}

\begin{abstract}
Ratio scaling was used to obtain from 5 subjects estimates of the subjective dissimilarity between the members of all possible pairs of 17 tactile surfaces. The stimuli were a diverse array of everyday surfaces, such as corduroy, sandpaper, and synthetic fur. The results were analyzed using the multidimensional scaling (MDS) program ALSCAL. There was substantial, but not complete, agreement across subjects in the spatial arrangement of perceived textures. Scree plots and multivariate analysis suggested that, for some subjects, a two-dimensional space was the optimal MDS solution, whereas for other subjects, a three-dimensional space was indicated. Subsequent to their dissimilarity scaling, subjects rated each stimulus on each of five adjective scales. Consistent with earlier research, two of these (rough/smooth and soft/hard) were robustly related to the space for all subjects. A third scale, sticky/slippery, was more variably related to the dissimilarity data: regressed into three-dimensional MDS space, it was angled steeply into the third dimension only for subjects whose scree plots favored a nonplanar solution. We conclude that the sticky/slippery dimension is perceptually weighted less than the rough/smooth and soft/hard dimensions, materially contributing to the structure of perceptual space only in some individuals.
\end{abstract}

In touch, as in other sensory modalities, stimuli can differ in a large number of physical properties. For example, the surfaces of objects presented to the sense of touch can differ from one another in their frictional resistance to lateral movement of a finger across them, in their compressibility in response to radial force, their thermal conductivity, and in the presence, density, size, composition, and arrangement of structural elements that disturb the flatness of the surface. Considerable research documents the close relationship between these properties and subjective qualities such as roughness, softness, and slipperiness (Connor, Hsiao, Phillips, \& Johnson, 1990; Connor \& Johnson, 1992; Katz, 1925/1989; Lederman \& Taylor, 1972; Srinivasan \& LaMotte, 1996; Srinivasan, Whitehouse, \& LaMotte, 1990; S. S. Stevens \& Harris, 1962; Taylor \& Lederman, 1975). When an overall impression of such a stimulus is obtained in a relatively brief exposure, it is reasonable to ask (1) which and how many of its properties enter into this impression, and how fully they are combined, and (2) how the stimuli seem to the subject to be related to one another-for example, whether they are arranged in an orderly dimensional structure (analogous, e.g., to color space) defined by the subjective dimensions corresponding to their component properties.

An important step in answering the first of these questions was taken by Klatzky, Lederman, and Reed (1989).

This work was supported by NSF Grant SBR-9514432. Correspondence should be addressed to M. Hollins, Department of Psychology, CB\# 3270, Davie Hall, University of North Carolina at Chapel Hill, Chapel Hill, NC 27599 (e-mail: mhollins@email.unc.edu).
They investigated subjects' ability to use multiple haptic dimensions by asking them to rapidly classify small, specially constructed objects that varied in hardness, surface texture (specifically roughness), and shape into categories to which they had been assigned by the experimenter. They found that subjects were substantially faster when the sorting could be based on two redundant (i.e., correlated) dimensions than when only one dimension was predictive. The speed gain was greatest for the roughness/ hardness combination, although there was some increase in speed when shape was (individually) combined with roughness and hardness. Surprisingly, however, there was no additional gain when all three dimensions were redundant. On the basis of this and other experiments, Klatzky et al. conclude that the roughness and hardness dimensions are "integral" (in Garner's, 1974, sense of the term) with respect to each other but that each of these dimensions has a somewhat more "separable" relationship with shape. In this and later studies (Lederman, Klatzky, \& Reed, 1993; Reed, 1994; Reed, Lederman, \& Klatzky, 1990), they examine the idea that compatibility of the exploratory procedures (Lederman \& Klatzky, 1987) used to obtain information about particular stimulus properties may help to determine the relationship between dimensions. Regardless of the factors responsible, however, the data of Klatzky et al. (1989) raise the possibility that there is a limit on the number of haptic dimensions that subjects can combine into an overall perceptual experience.

Multidimensional scaling (MDS) provides another way to examine the role of dimensions in the perceptual organization of sets of stimuli (Shiffman, Reynolds, \& 
Young, 1981). In this computationally intensive method, measurements of subjective dissimilarity are combined to yield a model of perceptual space in which points represent stimuli. The distance between any two points reflects as nearly as possible the perceptual differentness of the corresponding stimuli. Spaces of different dimensionalities can be computed and can be evaluated on several bases, including the degree to which interstimulus distances depart from the measures of subjective dissimilarity.

In an earlier MDS study of tactile texture perception (Hollins, Faldowski, Rao, \& Young, 1993), we asked subjects to sort 17 qualitatively different stimuli into three to seven categories based on perceptual similarity. The proportion of subjects who placed a given pair of textures into separate categories was used as a measure of perceptual dissimilarity. The data were analyzed assuming ordinal measurement, and candidate solutions of dimensionalities $1-5$ were computed. We had hoped that stress, a measure of the degree to which a solution fails to account for the data, would decline rapidly as dimensionality increased up to the "true" solution, beyond which there would be little additional decline. There was, however, no such elbow in the scree plot; instead, stress decreased gradually as solution dimensionality increased. Qualitative scrutiny of the solutions themselves nevertheless led us to favor the three-dimensional (3-D) space, which was therefore chosen for comparison with adjective scale ratings of the stimuli. We found that rough/smooth ratings were highly correlated with one dimension of the space, and soft/hard ratings were strongly correlated with a nearly orthogonal axis. None of the other three adjective scales on which ratings had been obtained (slippery/sticky, flat/bumpv, warm/cool) was highly correlated with the third dimension, however, leaving us uncertain as to its perceptual significance.

One factor hindering definitive interpretation of subjective texture space on the basis of the Hollins et al. (1993) study is that their data had to be combined across subjects in order to obtain a dissimilarity matrix. How well the resulting MDS solution describes individual subjects' perceptual organizations is unknown, and substantial differences between subjects could interact to produce a "group" space that is not representative of any individual. For example, if subjects have two-dimensional (2-D) perceptual structures that are qualitatively different from one person to another, the computational result would likely be an overall solution suggesting the presence of more than two dimensions.

The purpose of the present study was therefore to continue our investigation of tactile texture perception using MDS, but in a way that would permit us to gain insight into the perceptual organization of textures in individual subjects, using ratio scaling methods to obtain measures of dissimilarity. We hoped to answer four specific questions: (1) whether the perceptual organization of textures is well captured by a spatial, multidimensional metric;
(2) if so, what the dimensionality of this space is; (3) what the dimensions represent; and (4) whether there are significant individual differences in the way perceived textures are organized.

\section{METHOD}

\section{Subjects}

Four graduate students and 1 undergraduate served as subjects. They ranged in age from $2 \mathrm{l}$ to 26 years, and all were right-handed. None was aware of the specific questions addressed by the research, although $i$ (Subject A) was familiar with MDS. They were paid for their participation.

\section{Stimuli}

The stimuli were 17 surfaces of qualitatively different textures, each measuring $7.6 \times 4.4 \mathrm{~cm}$. Sixteen of the surfaces were glued onto $2.3-\mathrm{cm}$-thick Plexiglas blocks of the same size; the $17 \mathrm{th}$ was the smooth surface of another such block. The surfaces were (1) Plexiglas, (2) lightly sanded balsa wood, (3) unglazed tile, (4) rubber from a balloon, stretched flat across the surface of the block, (5) Styrofoam, (6) corduroy, its 3-mm wales oriented perpendicular to the long axis of the block, (7) 140-wt watercolor paper, (8) cellulose kitchen sponge, (9) cork composite, from a corkboard, (10) shag rug, (11) 3-mm-wide pieces of straw, running laterally and arranged in a contiguous series extending the length of the block, (12) synthetic fur, (13) nylon scouring pad, (14) synthetic suede, (15) silk, (16) felt, and (17) 220-grit aluminum oxide sandpaper. A number of these were similar to textures used by Hollins et al. (1993). As in the earlier study, we intentionally chose surfaces that gave rise to a wide range of tactile sensations and that differed physically from one another in a variety of ways.

\section{Procedure}

Dissimilarity scaling. After having the procedure explained and giving written informed consent, the subject sat at a table and extended his/her right arm under a curtain that prevented him/her from seeing the experimenter (seated opposite) and the stimuli. The subject's right arm was draped over a pillow, with the hand made into a fist with only the index finger extended, volar surface down. In presenting stimuli, the experimenter positioned a surface below the fingertip and then raised the surface until it contacted, and produced just visible upward movement of, the finger. The experimenter then pulled the surface horizontally so that it moved from proximal to distal along the subject's fingertip a distance of approximately $5 \mathrm{~cm}$, at a rate of about $2.5 \mathrm{~cm} / \mathrm{sec}$.

A trial consisted of the sequential presentation of two surfaces. After the second stimulus, a slip of paper on which a horizontal line was printed was presented to the subject, who was asked to make a mark on it with his/her left hand, using a pen. The line was a visual analog scale of the perceived differentness of the two textures presented on that trial. From its left termination (which was marked " 0 "), it ran $19.1 \mathrm{~cm}$ to the right edge of the paper. The subjects were asked to mark the line so that the distance from its left end to their mark would be proportional to the dissimilarity between the two textures.

Each of the five sessions began with a presentation of each of the 17 surfaces, in a random order that differed across sessions and across subjects. The subjects were not asked to respond in any way during this "familiarization" phase. After these initial presentations, experimental trials began. Stimulus pairs to be used on a trial were ordered randomly without replacement. In the first session, which was devoted to practice, 30 pairs were presented. This allowed the subjects to adjust their responses so that, in all cases, their mark would "fit" on the paper, while still constituting a ratio scale. All 
subjects reported at the end of the practice session that they had been able to do this. They were told that the horizontal line continued to the edge of the page to remind them that there might exist textures more different from one another than those being used in the experiment.

There are 136 pairwise combinations of 17 items, ignoring the order of the two items within a pair. In the second and third sessions combined, each of these combinations was presented once. Sixtyeight randomly chosen pairs of textures (selected separately for each subject) were presented during the second session, and the remaining 68 were presented during the third session. All possible pairs were again presented (in a new random order) in the fourth and fifth sessions combined, with the order of textures within each pair being the opposite of that used in the second and third sessions.

Adjective scaling. Following the completion of the dissimilarity measurements, the subjects returned to the laboratory for a final session in which they rated each of the 17 stimuli on each of five adjective scales. Each of these scales consisted of a $12.7-\mathrm{cm}$ horizontal line, labeled with an adjective at each end. This scale length was chosen on the basis of the recommendation of Shiffman et al. (1981) and to ensure comparability with our earlier study (Hollins et al., 1993).

Four of the scales were identical to ones used in the Hollins et al. (1993) study: rough/smooth, hard/soft, slippery/sticky, and warm/ cool. A fifth scale, flat/bumpy, was treated by the subjects of the earlier study as essentially synonymous with rough/smooth and was therefore not used in the present experiment. A new scale, moldable/springy, was introduced, however, to follow up the impression of Hollins et al. (1993) that some variance orthogonal to the rough/smooth-hard/soft plane of their 3-D MDS space appeared to reflect differences in springiness among textures. Springy was defined for the subjects as "elastic," with the additional comment that a springy stimulus "returns to its original shape when deformed by pressure." It was stressed that this quality was independent of the amount of pressure needed to produce that deformation. Moldable was defined as "inelastic": A moldable surface "does not return to its original shape when deformed by pressure."

The session began with a presentation of each of the 17 stimuli, to refamiliarize the subjects with them. The subjects did not respond to these presentations. The order of the stimuli during this initial phase was random and (as always) different for each subject. After this, the rating method was described to the subjects, and each of the adjectives defined. The stimuli were then presented again, in a new random order. After a stimulus had been presented, the subject was given an adjective scale and asked to mark it at a point representing the relative degree to which the two adjectives defining that scale were descriptive of the stimulus. Once the subject had responded, and the slip of paper bearing the scale was retrieved by the experimenter, the same surface was presented again and a new scale presented. This process was iterated until the surface had been rated on all five scales.

The order of the scales was always the same: springy/moldable, soft/hard, rough/smooth, sticky/slippery, and cool/warm. For 3 of the subjects, the first adjective of each pair was on the left end of the scale, and the opposite adjective was on the right end; for the other 2 subjects, the reverse was true. After a rating on each scale had been obtained for each surface, the subjects were given a 10 . min break, after which they repeated the process, but with the surfaces presented in a new random order. In addition, each adjective scale was now reversed, so (for example) a subject who had previously used a scale with rough on the left and smooth on the right now saw smooth on the left and rough on the right. This second set of ratings concluded the subject's participation.

\section{Data Analysis}

Deriving MDS solutions. Each dissimilarity-scaling response was scored by hand, the distance from the left $(0)$ end of the $19.1-\mathrm{cm}$ scale being estimated to the nearest $0.01 \mathrm{~cm}$ (a degree of nominal precision somewhat exceeding the accuracy of measurement). Since two ratings of each stimulus pair had been made by each subject, the subject's data were represented by two half-matrices. The program ALSCAL (SPSS version) was used to derive MDS solutions (Young \& Lewyckyj, 1979). In preliminary analyses, MDS solutions derived from a subject's two half-matrices appeared comparable. In the main analysis, therefore, the relationship between the two half-matrices was treated as unconditional (i.e., not sessiondependent).

Because the subjects had been instructed to mark on the visual analog scale in such a way that distance from the left end of the scale was directly proportional to perceived dissimilarity of the two textures being compared, an assumption of ratio measurement was used in determining the MDS solutions. The space to be computed was assumed to be Euclidean.

We derived for each subject solutions ranging from one to six dimensions, and examined plots of stress (i.e., the extent to which a solution fails to account for the variance in the data) as a function of dimensionality. In evaluating these scree plots, we looked for signs of an "elbow" indicating the presence of a dimensionality beyond which there was an abrupt attenuation in the ability of higher order solutions to capture additional variance; this is a time-honored, but not infallible, method of identifying the optimal solution (Schiffman et al., 1981).

Applying adjective scales. Each subject had given two ratings of each stimulus texture on each adjective scale. As explained previously, the left/right positions of the adjectives marking the ends of the scale were switched between its two presentations. To make a subject's two ratings of a given object on a particular scale comparable, distance to the subject's mark was always measured from the hard, moldable, smooth, slippery, or warm end of the scale. Because of the reasonably close agreement between the two ratings obtained in this way, they were averaged to yield a single value for each subject's rating of each object on each scale.

We used these values to carry out, for each subject separately, multivariate regression analyses to determine the extent to which the previously computed 1-, 2-, and 3-D MDS solutions predicted the adjective-scale ratings. This information was combined with the scree plots and other considerations to make a preliminary judgment as to the subjects' optimal MDS solutions.

We also examined the univariate regression of individual adjective scales onto the 2- and 3-D MDS solutions. In evaluating whether a given adjective scale might reflect a perceptual dimension used by the subject, we considered both the proportion of scale variance captured by the space and the significance of that proportion. Because two or more adjective scales might to some degree reflect the presence of the same underlying perceptual dimension, the correlations between adjective scales were also examined. By learning how the adjective scales were related to the dimensions of perceptual space and to each other, we hoped to identify and understand the optimal MDS solution.

\section{RESULTS}

\section{Quantitative Indicators of Dimensionality}

Stress. Scree plots for the 5 subjects are shown in Figure 1 . They show that stress tends in general to decline with increasing dimensionality: Up to a point, a solution with more degrees of freedom can typically capture more of the variance in the data. There is for every subject a large drop in stress between the 1- and 2-D solutions; beyond 3 dimensions, however, solutions of increasing order bring with them little, if any, reductions in stress. (The slight increase in stress at higher dimensionalities 


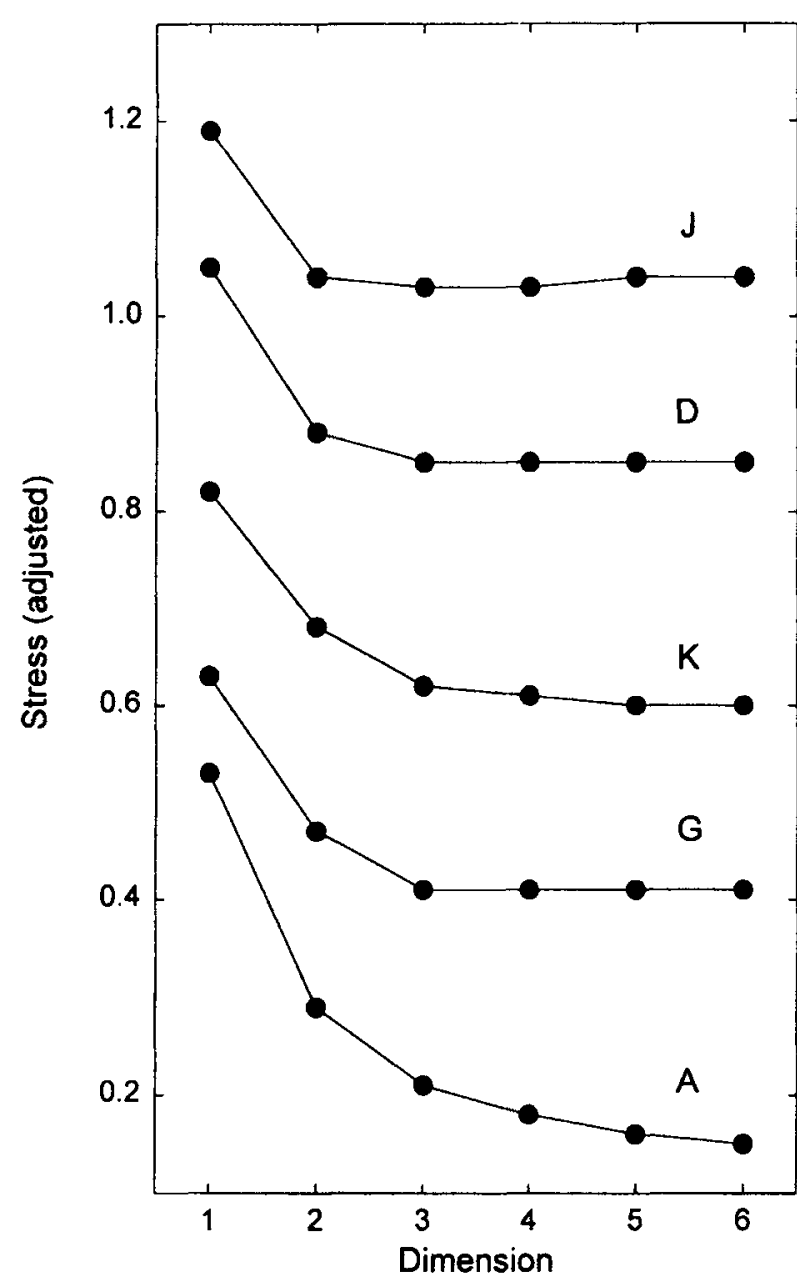

Figure 1. Stress of MDS solutions, plotted as a function of their dimensionality. The ordinate indicates unmodified stress values for Subject A; data of the other subjects have been shifted upward in increments of .2 for clarity. The scree plots are arranged from top to bottom in roughly increasing order of the degree to which they suggest the presence of three or more dimensions in the participant's perceptual space.

for Subjects D and $J$ may result from the fact that, while ALSCAL's algorithm is convergent [Shiffman et al., 1981, pp. 83-84], it seeks to minimize not stress but s-stress, a related quantity based on squared distances.)

Despite these similarities, there are clear individual differences among the scree plots. One of the subjects $(\mathrm{J})$ shows a pronounced elbow at the 2-D solution. Since there is little if any change in stress for further increases in solution dimensionality, parsimony argues that this solution is optimal. Subject $G$, on the other hand, shows a reasonably clear elbow at the 3-D solution, suggesting that for him this is optimal. The scree plots of Subjects D and $\mathrm{K}$ are intermediate in this regard: They indicate that either a 2-D or 3-D solution is optimal but lack a clear elbow that would serve as a basis for deciding between these possibilities. Finally, Subject A's scree plot asymptotes so gradually as to provide little evidence of an opti- mal dimensionality; beyond 3, however, further increases in dimensionality involve only modest improvements in fit.

In summary, these scree plots show marked individual differences, which (variously) suggest the appropriateness of either a 2-D or a 3-D solution, or something in between.

Multiple regression of adjective scales. To obtain a better understanding of the subjects' perceptual spaces, we examined the way in which the adjective-scale ratings mapped onto the MDS solutions, using regression analysis. The first question we asked was whether the perceptual structure uncovered by MDS was sufficient to account for the family of scales we used. To address this issue, we carried out, for each subject and dimensionality, a multivariate regression, with the coordinates of the principal dimensions of the MDS space serving as the independent variables and with the adjective-scale ratings serving as the five dependent variables.

The analysis yields a quantity, Wilks's lambda $(\Lambda)$, which is approximately the complement of the proportion of variance in the adjective-scale ratings that can be accounted for by an MDS solution (J. Stevens, 1996, pp. 191-192). For the l-D solutions, this proportion (i.e., $1-\Lambda)$ varied across subjects from .727 to $.926(M=$ $.828)$; for the 2-D solutions, it varied between .946 and $.996(M=.968)$; and for the 3-D solutions, it varied from .981 to $.997(M=.988)$. A caveat is in order: The quantity $1-\Lambda$ may somewhat overestimate the proportion of variance in the adjective ratings accounted for by the MDS solution, because it is based on observations that are not independent.

Nevertheless, the substantial increases in $1-\Lambda$ from the 1-D to the 2-D solutions imply that a plane does a considerably better job than a line does of "making room" for representation of the reported differences in roughness, hardness, and other scaled properties, among the stimuli. The values of $1-\Lambda$ for the $2-D$ solutions are in fact so close to unity that there is little room for them to increase further in the 3-D solutions. It is noteworthy, however, that this latter increase is greatest and the value of $1-\Lambda$ for the 2-D solution is lowest for the 2 subjects (A and $G$ ) whose scree plots most strongly suggest the existence of a third dimension. In summary, the multivariate analysis, when combined with results of the stress analysis, indicates that the adequacy of the 2-D solution may vary from one individual to another.

\section{Two-Dimensional Solutions}

Examination of stress is, however, only one way of evaluating dimensionalities and comparing the results of different individuals. Another and more direct way is to examine the MDS solutions themselves. We began this process by comparing the 2-D solutions of the 5 subjects. These showed a marked consistency in the overall arrangement of the surfaces within the solution space. Figure 2 shows two representative solutions: those for Subject $J$ (whose scree plot had an elbow at 2) and Sub- 
ject $G$ (for whom the elbow was at 3 ). Despite this difference in stress relationships, the arrangement of the 17 stimuli within the two solutions is remarkably the same: In both cases, for example, felt and suede are near the center of the space, with sandpaper displaced vertically (i.e., along MDS Dimension 2) from them; rug and fur are near each other in the upper left; straw is in the lower left; wood, rubber, glass, and other stimuli form a cluster on the right; and so on. The correspondence is by no means perfect: For example, corduroy and Styrofoam are close together for Subject G, but they are fairly widely separated for Subject J. Nevertheless, the overall picture is one of consistency.

This consistency is not surprising in view of the relatively strong correlations between subjects in dissimilarity ratings for the different pairs of stimuli. The (Pearson) correlation coefficients for pairs of subjects range from .66 to .80 , with a median of .77 ; all are highly significant $(p<.001)$. There is thus a strong element of agreement across subjects in the psychophysical data, even in cases where the scree plots suggest solutions of different dimensionalities.

The similarity of the 2-D solutions does not mean that these solutions are necessarily optimal; as will be seen later, the 3-D solutions also exhibit marked similarities. Nor is the degree of consistency so great as to rule out the possibility that, for some subjects, the underlying perceptual structure is in fact 2-D, while, for others, it is 3-D.

Position of adjective scales in 2-D solutions. We will now consider the way the adjective scales are arranged in 2-D MDS space, again using Subjects $\mathbf{J}$ and $\mathbf{G}$ as examples (Figure 2). The orientation and relative length of each line reflect the standardized coefficients of regression $(\beta)$ of that scale onto the dimensions of the space. Specifically, the line extends from the origin to a point defined by $2.5 \times \beta$ (for visual clarity); in addition, the line is extended into the opposite quadrant so that it is symmetrical about the origin. Standardized regression coefficients are used because the sum of their squares is approximately proportional to the fraction $\left(R^{2}\right)$ of variance in the adjective-scale ratings that is accounted for by the MDS space (Shiffman et al., 1981). Thus, the rough/ smooth line is relatively long in both plots because, for this adjective scale, $R^{2}$ was high both for Subject J (.95) and for Subject $\mathrm{G}(.76)$; the cool/warm line, in contrast, is relatively short because $R^{2}$ for this scale was smaller (.36 and .23 for Subjects J and $\mathrm{G}$, respectively).

The relative positions of the adjective scales in all subjects' 2-D MDS spaces will now be described, using the results for Subjects $\mathbf{J}$ and $\mathbf{G}$ (Figure 2) as a point of reference. For all 5 subjects, a large and highly significant $(p<.001)$ fraction of the variance on the rough/smooth scale was accounted for by the space ( $R^{2}$ range, .68-.95, $M=.80)$.

The MDS solution also accounted to a significant $(p<.05)$ degree for variance along the soft/hard scale, with $R^{2}$ ranging from .41 to $.93(M=.63)$. The large angle between the lines representing these two scales reflects the fact that, for all of the subjects but 1 (Subject A, $r=.70$ ), the soft/hard and rough/smooth scales were relatively uncorrelated ( $r$ ranged from -.06 to $.42, M=.20$ ).

Closely related to soft/hard was the springy/moldable scale, for which the space also accounted for a significant component of the variance in all subjects, with $R^{2}$ ranging from .40 to $.86(M=.59)$. These two scales were closely correlated in all subjects; however, the correlation differed in sign from one subject to another, being positive in $3(r \mathrm{~s}=.80, .86, .97)$ and negative in the other 2 $(r \mathrm{~s}=-.69,-.81)$. That is, 3 subjects (including J) interpreted springy as virtually equivalent in meaning to soft, whereas the other 2 (including G) used moldable in this sense. Clearly, our plan to capture a different aspect of texture-the degree to which a surface resumes its original shape when pressure is released, as distinct from the amount of pressure needed to deform it - was unsuccessful. The results nevertheless imply that the underlying perceptual dimension, whether called by its usual names (i.e., soft/hard) or by novel ones, is robust and readily lends itself to psychophysical scaling. Because the springy/moldable scale was ambiguous and was treated by the subjects as interchangeable with soft/hard, we have omitted it from the figures and make no further reference to it.

In contrast to the scales described so far, variance along the sticky/slippery scale was significantly accounted for by the 2-D MDS solution in only 3 of the 5 subjects $\left(R^{2}\right.$ range, .24-.63, $M=.45$ ). However, sticky/slippery and rough/smooth ratings were consistently correlated ( $r$ range, $.41-.85, M=.61$ ), a fact illustrated by the modest angle between the lines representing these scales in the figure. The results suggest that the subjects varied somewhat in the importance of this scale in their perceptual space and in the extent to which its meaning overlapped, for them, with the rough/smooth scale. The difference between the rough/smooth and sticky/slippery scales was not a haphazard one; their relative positions were always the same: rough fell between sticky and soft, albeit usually (except for Subject A) closer to the former.

Only a modest proportion (significant in 3 subjects) of the variance in cool/warm ratings was accounted for by the MDS space: $R^{2}$ ranged from .23 to $.60(M=.39)$. The $\mathrm{cool} /$ warm line's position relative to the other scales differed from subject to subject, being variously closest to sticky/slippery, soft/hard, or (for Subjects J and G, as shown in Figure 2) rough/smooth.

In summary, there are elements of consistency, but also evidence of individual differences, in the way adjective scales map onto the 2-D MDS space. The rough/ smooth and soft/hard scales are robust for all subjects, and, in all cases but one, the lines representing them in space are separated by a wide angle. Two other scales, sticky/slippery and cool/warm, vary considerably from subject to subject in their position within, and degree of relatedness to, the space. 


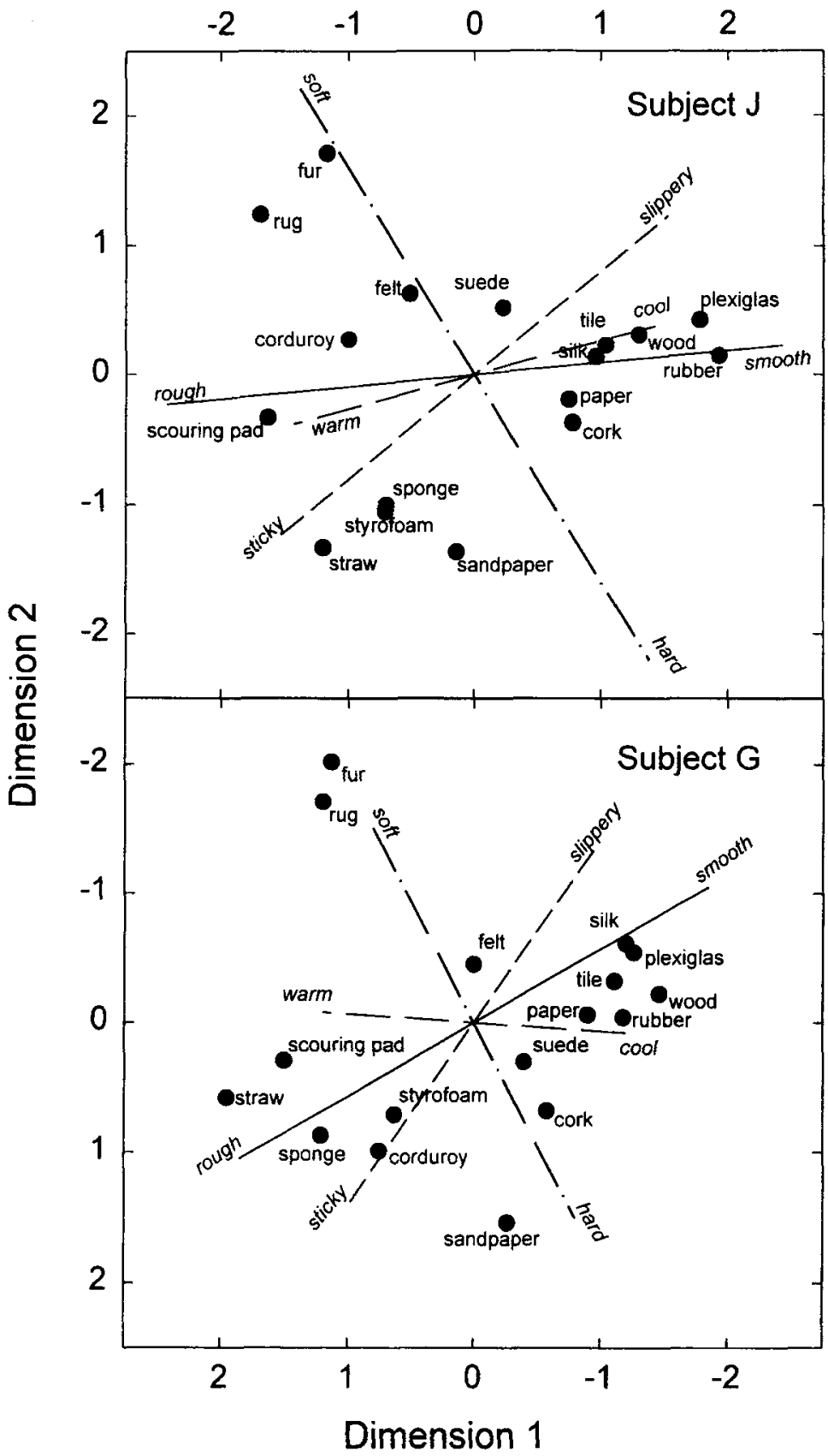

Figure 2. Two-dimensional MDS solutions for 2 representative subjects. Dimension polarity is essentially arbitrary; the polarity of Dimension 1 for Subject $G$ has been reversed to make the similarity of the solutions more apparent. Adjective scales are shown regressed into the space, their endpoints defined by \pm 2.5 times their adjusted regression coefficients $(\beta)$ in the 1 st and 2 nd dimensions.

\section{Three-Dimensional Solutions}

Representative 3-D solutions are shown for Subjects J and $\mathrm{G}$ in Figures 3 and 4 , respectively. The space is viewed from different directions in the two panels of each figure: It is viewed along Dimension 3 ("from above") in the upper panel and along Dimension 2 ("from the front") in the lower panel. The projections of these solutions onto the plane defined by Dimensions 1 and 2, shown in the upper panel, closely resemble the 2-D solutions shown in Figure 2. In both cases, for example, most of the points are arranged in a broad swath that parallels the rough/smooth axis; and in both cases, points representing the fur and the rug are located in the upper left, with the felt lying intermediate between them and 
the rough/smooth axis. A similar correspondence between the 2-D solution and the projection of the 3-D solution onto its first 2 dimensions prevailed for the other subjects as well.

The "front view" of the 3-D solutions, however, presents an unexpected but clearcut difference between the spaces of Subjects $J$ and $G$. For Subject $J$, the adjective scales all lie in the plane formed by Dimensions 1 and 2: No scale is angled up more than $10^{\circ}$ into the 3 rd dimension. For Subject $G$, on the other hand, most scales are again clustered in or near the horizontal plane, but onesticky/slippery - tilts up at $45^{\circ}$ into the 3 rd dimension. This is notable in view of the fact that $J$ was the subject whose scree plot most clearly favored the 2-D solution, whereas that for Subject $\mathrm{G}$ most clearly indicated the 3-D solution as optimal. These indications are now supported by these views of MDS space: Even though this solution is nominally 3-D, all of Subject J's adjective scales lie essentially in a plane, whereas for Subject $G$, a specific adjective scale makes use of the 3 rd dimension. Other subjects (not shown) display a pattern that is similar to that of either Subject J or Subject G. Thus, the 3-D solutions for Subjects D and $K$ are, like that of Subject $J$, roughly planar, although, in both of their cases, the plane in which the adjective scales lie is tilted slightly with respect to the Dimension 1/Dimension 2 plane, and there is more "jitter" of the adjective scales with respect to one another. For Subject A, on the other hand, whose scree plot appeared to suggest solutions of Dimensionality 3 or greater, the sticky/slippery scale was angled into the 3rd dimension at a substantial $38^{\circ}$ and was in fact nearly perpendicular to the plane formed by the rough/smooth and soft/hard axes. Consistent with these findings, the correlation of the sticky/slippery scale with the 3 rd dimension of MDS space was small or negligible in Subjects $\mathrm{J}, \mathrm{D}$, and $\mathrm{K}(r \mathrm{~s}=$ $.05, .25$, and -.11 , respectively) but was moderate in Subjects $\mathrm{G}$ and $\mathrm{A}(\mathrm{r} \mathrm{s}=-.58$ and .51 , respectively).

Table 1 shows $R^{2}$, the proportion of variance in each adjective scale that is accounted for by the 2- and 3-D MDS solutions. In most, but not all, cases, the 3-D solution accounted for somewhat more of the variance. It is of some interest that, for every subject, exactly three of the adjective scales were significantly $(p<.05)$ related to the 3-D MDS solution. These were the rough/smooth, soft/hard, and slippery/sticky scales for Subjects A, G, and $\mathrm{J}$ and the rough/smooth, soft/hard, and cool/warm scales for Subjects D and $\mathrm{K}$. Their degrees of correlation with one another determined whether the three significant scales all lay in roughly the same plane (Subjects D, $\mathrm{J}$, and $\mathrm{K}$ ) or one extended substantially into the $3 \mathrm{rd}$ dimension (Subjects $\mathrm{G}$ and $\mathrm{A}$ ). It is puzzling that both the sticky/slippery scale and the cool/warm scale were shown to be potentially viable, in that each was used by some of the subjects, but that no subject's MDS space reflects the use of both of these scales to a significant degree. Moreover, the same scales in the same subjects were "already" significant in the 2-D solutions, and, in fact, one scale that was significantly related to the 2-D solution (Subject J's $\mathrm{cool} /$ warm scale) was no longer so in the 3-D solution.

Subject A's 4-D solution did not reveal the use of any additional subjective continua by the subject. The 4 th dimension appeared to serve only as a vehicle for additional distancing of a few objects - particularly the rug and the balloon-from all others.

\section{DISCUSSION}

Several findings emerge from this investigation. The first is that there is remarkable consistency across subjects in the pattern of their estimates of the dissimilarity of tactile textures-a result that validates earlier findings based on group data. A second finding is that, despite these similarities, there are clear differences between subjects in the arrangement of textured surfaces and adjective scales within perceptual space, particularly with regard to whether a 3-D, as opposed to a 2-D, space is needed to optimally model their dissimilarity ratings. Third, while the two major dimensions of texture space are rough/smooth and soft/hard, as expected on the basis of earlier work, it is the sticky/slippery axis that is most consistently tilted into the $3 \mathrm{rd}$ dimension. Let us consider each of these findings in turn.

The 5 subjects were in considerable agreement regarding the relative similarity of different pairs of surfaces, as shown by the fact that the correlation between subjects was in no case less than .66. These high correlations are striking in view of the fact that the stimuli were extremely diverse everyday surfaces that were physically different from one another in many ways. They did not form ordered series or fall neatly into a small number of categories. Moreover, the subjects (none of whom had knowledge of the stimuli or of the specific questions being investigated) varied widely in their familiarity with scaling techniques. They ranged from an undergraduate chemistry student with no background in psychology (Subject G) to a graduate student with extensive knowledge of the mathematics of scaling (Subject A). It was perhaps the latter's sophistication and the effort he put into the task that made his results somewhat distinctive, as described earlier. Nevertheless, even his dissimilarity ratings were strongly correlated with those of the other subjects ( $r$ ranging from .66 to .76 ). The relative arrangement of the adjective scales was also similar in some respects from one subject to the next. The considerable degree of consistency of results across individuals shows that the perceptual organization of tactile textures is not idiosyncratic or haphazard but follows, in the main, systematic rules.

For most subjects, the nearly independent dimensions of rough/smooth and soft/hard dominate the 2-D MDS solution, as well as the Dimension 1/Dimension 2 plane of the 3-D solution. The robustness of these scales for every subject confirms the traditional view (initiated by Katz, 1925/1989) that these are the two major textural dimensions, as the results of our earlier study (e.g., Hollins et al., 


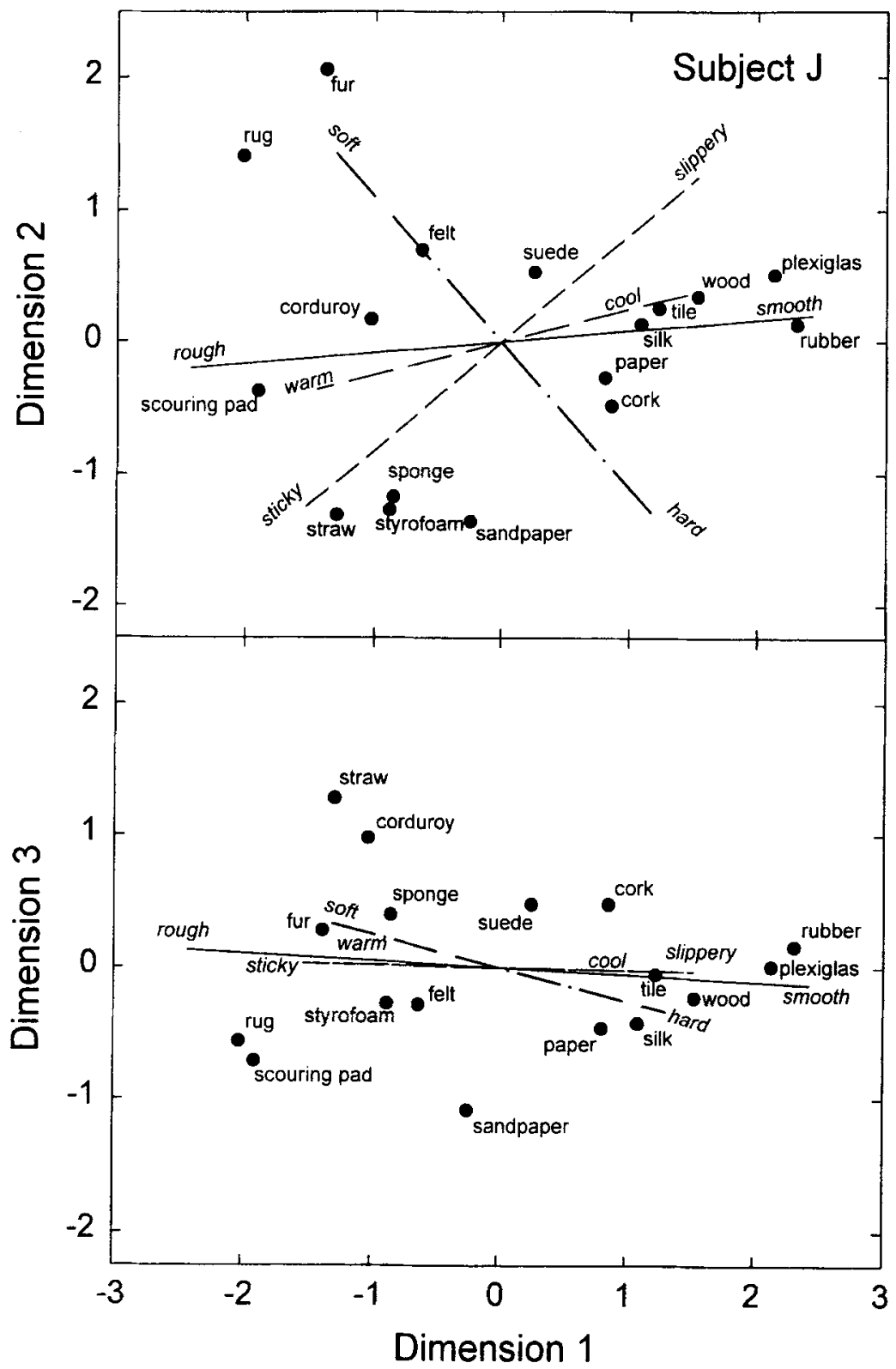

Figure 3. Three-dimensional solution for Subject $\mathbf{J}$, whose scree plot suggested that a 2-D solution was adequate. The upper plot shows the view of the space "along" Dimension 3 (i.e., from above); the lower plot shows a front view of the space. Adjective scales are shown regressed into the space, with endpoints defined by \pm 2.5 times their regression coefficients in each dimension.

1993) suggest. The fact that a Euclidean MDS space-reflecting in large part the presence of these two perceptual dimensions - is achieved with only moderate stress is consistent with the idea that rough/smooth and soft/hard are largely integral dimensions (Garner, 1974; Shepard, 1991), as Klatzky et al. (1989) first showed them to be using speeded-sorting paradigms.

Despite the overall consistency of results across subjects, one important individual difference emerged. For 3 subjects, the adjective scales are all roughly coplanar, with none tilting more than $30^{\circ}$ into the 3 rd dimension of the 3-D MDS solution. For the 2 other subjects, however, the sticky/slippery scale does so. Perhaps not surprisingly, these are the 2 subjects whose scree plots suggest the presence of a 3 rd dimension and for whom multivariate regression of the adjective scales showed the largest decline in Wilks's lambda between the 2-D solution and the 3-D one. Thus, when the optimal MDS solution is 3-D, the sticky/slippery scale is associated with the 3rd dimension. This association occurs despite the fact that, for 


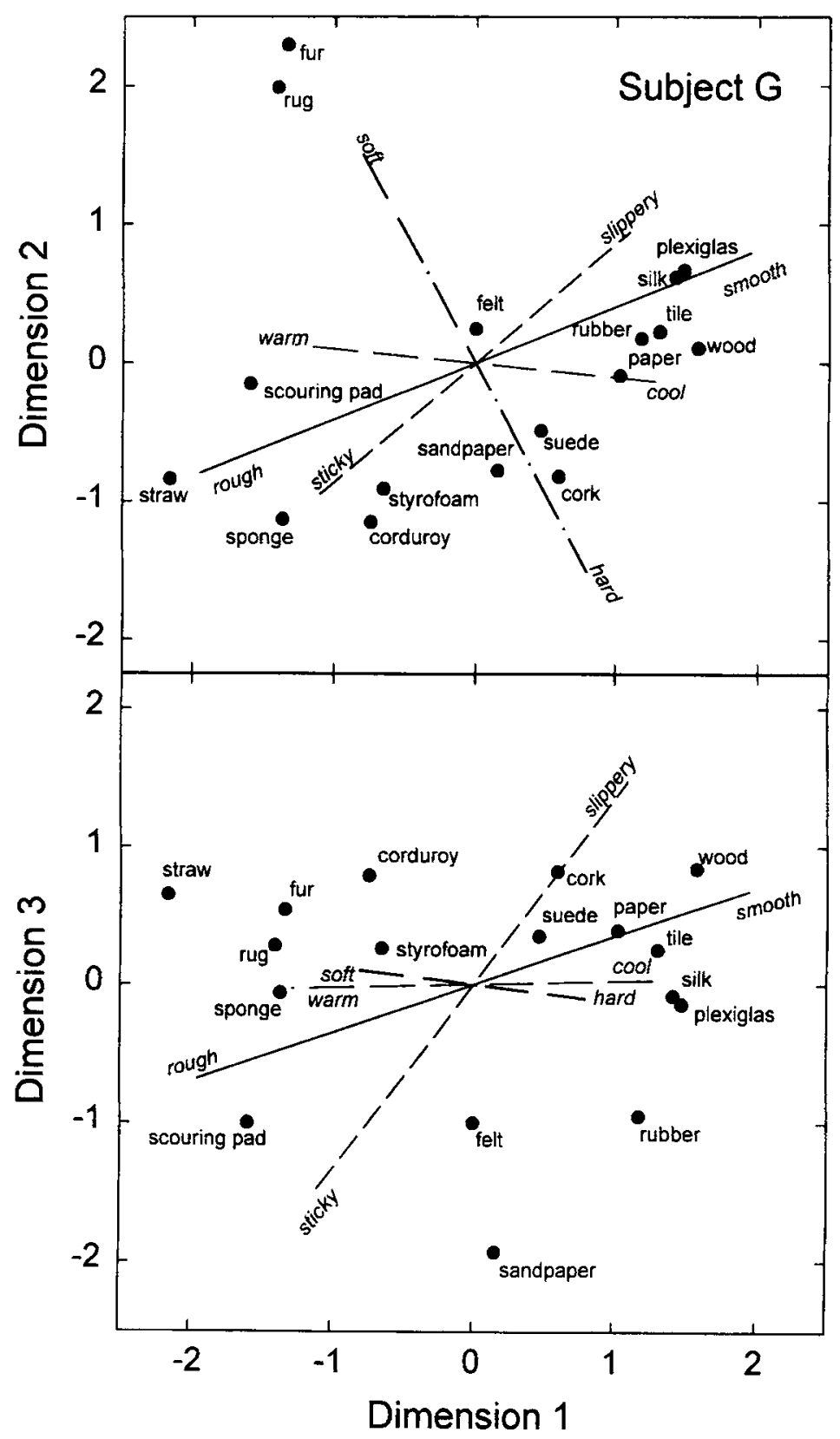

Figure 4. Three-dimensional solution for Subject G, whose scree plot suggested that this was the optimal solution. The upper plot shows the view of the space "along" Dimension 3 (i.e., from above); the lower plot shows a front view of the space. Adjective scales are shown regressed into the space, with endpoints defined by \pm 2.5 times their regression coefficients in each dimension.

all of the subjects, sticky/slippery ratings were at least moderately correlated with rough/smooth ratings. Perhaps this correlation reflects in part a statistical association, within our stimulus set, of the stimulus properties associated with these perceptual dimensions; such an interpretation is consistent with the fact that the roughness of machined gratings is not affected by friction (Taylor \& Lederman, 1975), the physical correlate of stickiness. It is likely, however, that the correlation also indicates some overlap in the meaning of the two scales as used by our subjects.

Whether it reflects similarity of percept, semantic overlap, or merely statistical association, the correlation of these two scales compromises the ability of stickiness/ slipperiness to constitute a fully independent dimension. Our overall conclusion is that texture space is dominated 
Table 1

Proportion of Adjective-Scale Variance ( $\left.\boldsymbol{R}^{2}\right)$ Captured by the Two-Dimensional and Three-Dimensional MDS Solutions

\begin{tabular}{|c|c|c|c|c|c|c|c|c|}
\hline \multirow[b]{2}{*}{ Subject } & \multicolumn{4}{|c|}{ 2-D Solution } & \multicolumn{4}{|c|}{ 3-D Solution } \\
\hline & $\begin{array}{l}\text { Rough/ } \\
\text { Smooth }\end{array}$ & $\begin{array}{l}\text { Soft/ } \\
\text { Hard }\end{array}$ & $\begin{array}{c}\text { Sticky/ } \\
\text { Slippery }\end{array}$ & $\begin{array}{l}\text { Cool/ } \\
\text { Warm }\end{array}$ & $\begin{array}{l}\text { Rough/ } \\
\text { Smooth }\end{array}$ & $\begin{array}{l}\text { Soft/ } \\
\text { Hard }\end{array}$ & $\begin{array}{c}\text { Sticky/ } \\
\text { Slippery }\end{array}$ & $\begin{array}{l}\text { Cool } \\
\text { Warm }\end{array}$ \\
\hline $\mathfrak{J}$ & $.947 * *$ & $.613 * *$ & $.633^{* *}$ & $.358^{*}$ & $.951 * *$ & $.610^{* *}$ & $.637 * *$ & .375 \\
\hline $\mathrm{D}$ & $.841 * *$ & $.738^{* *}$ & .304 & $.519 * *$ & $.891 * *$ & $.765^{* *}$ & .409 & $.526^{*}$ \\
\hline$K$ & $.765^{* *}$ & $.931^{* *}$ & .237 & $.603^{* *}$ & $.723^{* *}$ & $.922^{* *}$ & .164 & $.734 * *$ \\
\hline$G$ & $.758 * *$ & $.459^{*}$ & $.479 *$ & .233 & $.789 * *$ & $.446^{*}$ & $.694^{* *}$ & .257 \\
\hline A & $.682 * *$ & $.412 *$ & $.586 * *$ & .240 & $871 * *$ & $.559^{*}$ & $.655^{* *}$ & .310 \\
\hline
\end{tabular}

by two robust perceptual dimensions, rough/smooth and soft/hard, with a third, relatively weak dimensionsticky/slippery-manifesting itself in some individuals. The idea that haptic dimensions may differ in their prominence is not new: Reed et al. (1990) have found that when subjects briefly examine small objects differing in a number of ways, size appears te be more heavily weighted than other properties.

It is possible, of course, that the prominence of some perceptual dimensions relative to others (or even the presence of some and the absence of others) in the present study may be a reflection of the particular surfaces used as stimuli or the choice of scales on which the subjects were asked to give ratings; only further research can adequately determine the generalizability of the present findings. However, the importance of the rough/smooth and soft/hard dimensions and the presence of marked individual differences are findings that seem likely to transcend the limitations of the present study.

One remaining puzzle in the data is that, even for those 3 subjects whose adjective scales are roughly coplanar, some of the individual textures are consistently displaced in the 3 rd dimension with respect to others. For example, the corduroy is, for all 3 subjects (in fact for all 5), one of the farthest displaced from the Dimension 1/Dimension 2 plane, whereas the sandpaper and the scouring pad are among the farthest displaced from the plane in the opposite direction. Yet no obvious perceptual continuum appears to explain the overall disposition of textures in the 3 rd dimension for subjects $\mathrm{J}, \mathrm{D}$, and $\mathrm{K}$. We are inclined to suspect that at least some of the distancing of objects from one another in the 3 rd dimension reflects their individual distinctiveness rather than their position along a scale. For example, the corduroy was quite distinctive (and spontaneously recognized by several of the subjects, according to their subsequent anecdotal reports) by virtue of its ridges; the sandpaper and scouring pad were distinctive by virtue of their abrasiveness.

These distinctive, recognizable qualities are what Katz (1925/1989) referred to as Spezifikationen (identifying characteristics), as opposed to Modifikationen (dimensional properties). His writings leave unanswered the question of whether identifying characteristics are simply particular, learned combinations of values on dimen- sional scales or whether they lie outside of dimensional space. We are inclined to believe that the truth lies between these two extremes. In principle, any quality can be made into a dimension: For example, it would be possible to create a family of textures in which the presence of the ridged property of corduroy was systematically varied. Yet this hypothetical dimension does not appear to be one of the major dimensions in terms of which a diverse array of textures is spontaneously organized by the perceiver. The number of potential dimensions that subjects could use (and perhaps would, under carefully arranged experimental conditions) to structure their perceptions is very large, but subjects appear to be biased to attend to only two or three of these when presented with a relatively unstructured stimulus set.

We suspect, then, that subjects' dissimilarity ratings may to some degree reflect the distinctiveness or recognizability of particular stimuli, in addition to their dimensional properties. This was the conclusion reached by Hollins et al. (1993) in examining the MDS solutions of more than 3 dimensions: These supernumerary dimensions appeared to serve only to allow particular, distinctive surfaces to distance themselves from all other stimuli. In the one case (Subject A) where the presence of a 4th dimension seemed, on the basis of his scree plot, to be a possibility in the present study, the 4th dimension enabled the highly distinctive shag rug to become remote from all other stimuli. In these situations, distinctiveness is manifested spatially within the MDS solutions, but, in doing so, it probably sheds no light on dimensional structure per se. We believe the same may apply to some of the dispersion of stimuli even within MDS solutions of lower (and fundamentally valid) dimensionality.

Garner (1974) has found that when individual stimuli are meaningful (e.g., words), so that they are amenable to higher order cognitive processing, the dimensional structure in which they would otherwise be imbedded is weakened. A similar effect may occur when tactile textures are recognizable: The higher order processing that constitutes recognition may complicate attempts to examine the underlying dimensional structure of perceptual space. A full understanding of tactile texture perception will clearly require some systematic way of analyzing the responses to distinctive stimuli. 


\section{REFERENCES}

Connor, C. E., Hsiao, S. S., Phillips, J. R., \& Johnson, K. O. (1990), Tactile roughness: Neural codes that account for psychophysical magnitude estimates. Journal of Neuroscience, 10, 3823-3836.

CONNOR, C. E., \& JoHNSON, K. O. (1992). Neural coding of tactile texture: Comparison of spatial and temporal mechanisms for roughness perception. Journal of Neuroscience, 12, 3414-3426.

GARNER, W. R. (1974). The processing of information and structure. New York: Wiley.

Hollins, M., Faldowski, R., Rao, S., \& Young, F. (1993). Perceptual dimensions of tactile surface texture: A multidimensional scaling analysis. Perception \& Psychophysics, 54, 697-705.

KATZ, D. (1989). The world of touch (L. E. Krueger, Ed. and Trans.). Hillsdale, NJ: Erlbaum. (Original work published 1925)

Klatzky, R. L., Lederman, S. J., \& ReED, C. L. (1989). Haptic integration of object properties: Texture, hardness, and planar contour. Journal of Experimental Psychology: Human Perception \& Performance, 15, 45-57.

Lederman, S. J., \& KlatzKy, R. L. (1987). Hand movements: A window into haptic object recognition. Cognitive Psychology, 19, 342 368.

Lederman, S. J., Klatzky, R. L., \& Reed, C. L. (1993). Constraints on haptic integration of spatially shared object dimensions. Perception, 22, 723-743.

Lederman, S. J., \& TAYlor, M. M. (1972). Fingertip force, surface geometry, and the perception of roughness by active touch. Perception \& Psychophysics, 12, 401-408.

REED, C. L. (1994). Perceptual dependence for shape and texture during haptic processing. Perception, 23, 349-366.
Reed, C. L., Lederman, S. J., \& Klatzky, R. L. (1990). Haptic integration of planar size with hardness, texture, and planar contour. Canadian Journal of Psychology, 44, 522-545.

SHEPARD, R. N. (1991). Integrality versus separability of stimulus dimensions: From an early convergence of evidence to a proposed theoretical basis. In G. R. Lockhead \& J. R. Pomerantz (Eds.), The perception of structure: Essuys in honor of Wendell R. Garner (pp. 53-71). Washington, DC: American Psychological Association.

Shiffman, S. S., Reynolds, M. L., \& Young, F. W. (1981). Handbook of multidimensional scaling. New York: Academic Press.

Srinivasan, M. A., \& LaMotTe, R. H. (1996). Tactual discrimination of softness: Abilities and mechanisms. In O. Franzén, R. Johansson, \& L. Terenius (Eds.), Somesthesis and the neurobiology of the somatosensory cortex (pp. 123-135). Basel: Birkhäuser.

Srinivasan, M. A., Whitehouse, J. M., \& LaMotte, R. H. (1990). Tactile detection of slip: Surface microgeometry and peripheral neural codes. Journal of Neurophysiology, 63, 1323-1332.

STEVENS, J. (1996). Applied multivariate statistics for the social sciences (3rd ed.). Mahwah, NJ: Erlbaum.

Stevens, S. S.. \& Harris, J. R. (1962). The scaling of subjective roughness and smoothness. Journal of Experimental Psychology, 64, 489-494.

Taylor, M. M., \& Lederman, S. J. (1975). Tactile roughness of grooved surfaces: A model and the effect of friction. Perception \& Psychophysics, 17, 23-36.

YounG, F. W., \& LEWYCKYJ, R. (1979). ALSCAL user is guide (3rd ed.). Chapel Hill, NC: Data Analysis and Theory Associates.

(Manuscript received April 20, 1999. revision accepted for publication March 13, 2000.) 